\title{
NONQUADRATIC COST AND NONLINEAR FEEDBACK CONTROL
}

\author{
DENNIS S. BERNSTEIN \\ Department of Aerospace Engineering, The University of Michigan, Ann Arbor, MI 48109-2140, U.S.A.
}

\begin{abstract}
SUMMARY
Nonlinear controllers offer significant advantages over linear controllers in a variety of circumstances. Hence there has been significant interest in extending linear-quadratic synthesis to nonlinear-nonquadratic problems. The purpose of this paper is to review the current status of such efforts and to present, in a simplified and tutorial manner, some of the basic ideas underlying these results. Our approach focuses on the role of the Lyapunov function in guaranteeing stability for autonomous systems on an infinite horizon. Sufficient conditions for optimality are given in a form that corresponds to a steady-state version of the Hamilton-Jacobi-Bellman equation. These results are used to provide a simplified derivation of the nonlinear feedback controller obtained by Bass and Webber $(1966)^{38}$ and to obtain a deterministic variation of the stochastic nonlinear feedback controller developed by Speyer (1976). ${ }^{45}$
\end{abstract}

KEY WORDS Hamilton-Jacobi-Bellman equation Lyapunov function

\section{INTRODUCTION}

Linear-quadratic (LQ) control theory has been extensively developed over the past thirty years. In its most fundamental form, linear-quadratic control is based upon the following assumptions:

(i) the state dynamics and measurement equation are linear in both the state and control variables,

(ii) the performance measure to be minimized is quadratic in the state and control variables,

(iii) the plant disturbances and measurement noise are additive Gaussian white noise signals.

In addition to these explicit assumptions the following implicit assumptions are crucial:

(iv) the plant model is completely accurate,

(v) the state and control variables are not constrained.

Under these assumptions it is well known that the optimal feedback controller is linear. ${ }^{1}$

In many practical situations, however, one or more of these assumptions may be violated. For example, the state and measurement equations may be nonlinear, the performance measure may be nonquadratic, the disturbances may be nongaussian or nonadditive, the plant

Supported in part by the Air Force Office of Scientific Research under contracts F49620-89-C-0011 and F49620-89-C-0029.

This paper was recommended for publication by editor $M$. Green

$1049-8923 / 93 / 030211-19 \$ 14.50$

(c) 1993 by John Wiley \& Sons, Ltd.

Received 18 June 1991

Revised June 1992 
model may be uncertain, or the state and control variables may be constrained. In such cases there is no reason to expect that the optimal controller is linear. Rather, it should be expected that nonlinear controllers will have better performance than the best linear controllers. For example, if the plant is nonlinear then nonlinear controllers can be used to account for the global behaviour of the plant. ${ }^{2,3}$ Similarly, controllers designed for multiple plant linearizations are also nonlinear. ${ }^{4-6}$

In the case of optimal $H_{\infty}$ performance or robust stabilizability in the presence of unstructured plant uncertainty, nonlinear controllers offer no advantage over linear controllers. ${ }^{7,8}$ However, if the plant uncertainty is structured and if a quadratic Lyapunov function is assumed, then discontinuous nonlinear controllers have been shown to offer advantages over linear controllers. ${ }^{9-13}$ Continuous approximations to the discontinuous controllers of Reference 9 have been developed in References 14 and 15. Discontinuous controllers are also the focus of variable structure control which also addresses the problem of plant uncertainty. ${ }^{16-19}$ It is also shown in Reference 20 that nonlinear controllers can provide disturbance attenuation that is, in a certain sense, better than the performance of the optimal $H_{\infty}$ controller.

Adaptive controllers can be viewed as nonlinear controllers that operate in the presence of significant plant uncertainty. Such controllers have been shown to stabilize uncertain systems that cannot be stabilized by means of linear controllers. ${ }^{21-24}$

With regard to state and control constraints, one of the most common nonlinearities arising in applications is actuator saturation. ${ }^{25-32}$ Linear-quadratic and $H_{\infty}$ techniques, however, can, at best, only impose bounds on the mean-square values or $L_{2}$ norms of the state and control variables. Enforcing constraints such as $\|x(t)\| \leqslant \alpha,\|u(t)\| \leqslant \beta$ pointwise in time ( $L_{\infty}$ constraints) or $\int_{0}^{t_{1}}\|u(s)\| \mathrm{d} s \leqslant \gamma$ ( $L_{1}$ constraints) usually requires nonlinear controllers. Alternatively, it may be possible to design linear controllers that minimize the induced norm from $L_{2}$ disturbances to $L_{\infty}$ response signals thus minimizing peak output amplitude. ${ }^{33,34}$

In view of the advantages of nonlinear controllers over linear controllers, it is not surprising that significant effort has been devoted to developing a theory of optimal nonlinear regulation. References 35-58 comprise a representative, but not exhaustive, collection of papers in this direction. Our point of view is to regard this body of work as the foundation for a nonlinear-nonquadratic control framework that is analogous to linear-quadratic theory. To emphasize these connections and to provide the basis for future extensions, the goal of the present paper is to provide a tutorial exposition of a simplified framework for optimal nonlinear regulation in feedback control problems involving nonquadratic cost functionals. For simplicity and in accordance with practical motivation, we restrict our attention to time-invariant systems on the infinite interval. In this case asymptotic stability of the closedloop system is guaranteed by means of a Lyapunov function which plays a central role in the structure of the optimal nonlinear feedback control law. Furthermore, the Lyapunov function can clearly be seen to be the solution to the steady-state form of the HamiltonJacobi-Bellman equation.

We begin in Section 2 by considering a nonlinear controlled system with a performance functional evaluated over $[0, \infty)$. The performance functional is then evaluated in terms of a Lyapunov function that guarantees asymptotic stability (Lemma 2.1). This result is then specialized to the linear-quadratic case as well as to a multilinear setting. In Section 3 we consider a notion of optimality that is directly related to a given Lyapunov function. Although these controllers do not minimize a performance functional, they provide a direct connection to the requirement of asymptotic stability. In Section 4 an optimal control problem is stated 
and sufficient conditions (Theorem 3.1) are used to characterize an optimal nonlinear feedback controller. This result is then used to derive the nonlinear feedback controller obtained by Bass and Webber in Reference 38 as well as a deterministic variation of the stochastic nonlinear feedback controller developed by Speyer in Reference 45 . The desire to clarify and unify the results of References 38 and 45 is the main goal of the paper.

In future research, we intend to apply these results to the problem of fixed-structure nonlinear controller synthesis. To address this problem, the structure of the nonquadratic Lyapunov function, nonquadratic cost functional, and nonlinear feedback controller are fixed while the performance is optimized with respect to the controller gains. In this case the structure of the Lyapunov function can be viewed as providing the framework for controller synthesis by guaranteeing local or global asymptotic stability for a class of feedback controllers. The actual controller chosen for implementation can thus be the member of this candidate class that minimizes the given performance functional. In linear-quadratic regulator theory, for example, the Lyapunov function is the familiar quadratic functional $V(x)=x^{\mathrm{T}} P x$, while the gains for the linear feedback control are chosen to minimize a quadratic performance functional. In summary, the key idea is to use Lyapunov function theory to provide the framework and optimization to specify the gains.

\section{NONQUADRATIC COST EVALUATION}

In this section we investigate the role of Lyapunov functions in evaluating nonquadratic cost functionals. To expand upon the linear-quadratic case, we consider the problem of evaluating a nonquadratic cost functional depending upon a nonlinear differential equation. It turns out that the cost functional can be evaluated in convenient form so long as the cost functional is related in a specific way to an underlying Lyapunov function. Note that the results of this section make no explicit reference to control.

For simplicity we restrict our attention to time-invariant systems on the infinite horizon. Furthermore, for convenience we shall assume that existence and uniqueness properties of the given differential equations are satisfied. For the following result, let $\mathscr{D} \subset \mathbb{R}^{n}$ be an open set, assume $0 \in \mathscr{D}$, let $f: \mathscr{D} \rightarrow \mathbb{R}^{n}, L: \mathscr{D} \rightarrow \mathbb{R}$, and assume $f(0)=0$. Let ( ()' denote Frechét derivative.

\section{Lemma 2.1}

Consider the system

$$
\dot{x}(t)=f(x(t)), \quad x(0)=x_{0}, \quad t \geqslant 0,
$$

with performance functional

$$
J\left(x_{0}\right) \triangleq \int_{0}^{\infty} L(x(t)) \mathrm{d} t .
$$

Furthermore, assume there exists a $C^{1}$ function $V: \mathscr{D} \rightarrow \mathbb{R}$ such that

$$
\begin{aligned}
& V(0)=0 \text {, } \\
& V(x)>0, \quad x \in \mathscr{D}, \quad x \neq 0, \\
& V^{\prime}(x) f(x)<0, \quad x \in \mathscr{D}, \quad x \neq 0, \\
& L(x)+V^{\prime}(x) f(x)=0, \quad x \in \mathscr{D} \text {. }
\end{aligned}
$$


Then $x(t)=0, t \geqslant 0$, is a locally asymptotically stable solution to (1), and

$$
J\left(x_{0}\right)=V\left(x_{0}\right), \quad x_{0} \in \mathscr{D} .
$$

Proof. Let $x(t), t \geqslant 0$, satisfy (1). Then

$$
\dot{V}(x(t)) \triangleq \frac{\mathrm{d}}{\mathrm{d} t} V(x(t))=V^{\prime}(x(t)) f(x(t)), \quad t \geqslant 0 .
$$

Hence it follows from (5) that

$$
\dot{V}(x(t))<0, \quad t \geqslant 0, \quad x(t) \neq 0 .
$$

Thus, from (3), (4), and (9) it follows that $V(\cdot)$ is a Lyapunov function for (1), ${ }^{59}$ which proves local asymptotic stability of the solution $x(t)=0, t \geqslant 0$. Consequently, $x(t) \rightarrow 0$ as $t \rightarrow \infty$ for all initial conditions $x_{0} \in \mathscr{D}$. Now (8) implies that

$$
0=-\dot{V}(x(t))+V^{\prime}(x(t)) f(x(t)), \quad t \geqslant 0,
$$

and hence, by (6),

$$
\begin{aligned}
L(x(t)) & =\dot{V}(x(t))+L(x(t))+V^{\prime}(x(t) f(x(t)) . \\
& =-\dot{V}(x(t)) .
\end{aligned}
$$

Integrating over $[0, t)$ yields

$$
\int_{0}^{t} L(x(s)) \mathrm{d} s=-V(x(t))+V\left(x_{0}\right)
$$

Now letting $t \rightarrow \infty$ and noting that $V(x(t)) \rightarrow 0$ for all $x_{0} \in \mathscr{D}$ yields (7).

The main feature of Lemma 2.1 is the role played by the Lyapunov function $V(x)$ both in guaranteeing stability and in evaluating the cost functional $J\left(x_{0}\right)$. Note that condition (5) is equivalent to the fact that $(\mathrm{d} / \mathrm{d} t) V(x(t))<0$, where $x(t)$ satisfies (1). We prefer to write this condition in the form (5) as a 'static' condition on $x \in \mathscr{D}$ to avoid dependence on $t$.

Let us illustrate Lemma 2.1 with a familiar example. Consider the linear system

$$
\dot{x}(t)=A x(t), \quad x(0)=x_{0},
$$

with cost functional

$$
J\left(x_{0}\right)=\int_{0}^{\infty} x^{\mathrm{T}} R x \mathrm{~d} t
$$

where $R \in \mathbb{R}^{n \times n}$ is (symmetric) positive definite, $L(x)=x^{\mathrm{T}} R x$, and $\mathscr{D}=\mathbb{R}^{n}$. If $A$ is asymptotically stable, that is, has eigenvalues only in the open left half-plane, then there exists a positive-definite matrix $P \in \mathbb{R}^{n \times n}$ satisfying

$$
0=A^{\mathrm{T}} P+P A+R \text {. }
$$

Now define

$$
V(x)=x^{\mathrm{T}} P x
$$

which satisfies (3) and (4). Furthermore, with $f(x)=A x$ and $L(x)=x^{\mathrm{T}} R x$ it follows that

$$
V^{\prime}(x) f(x)=2 x^{\mathrm{T}} P A x=x^{\mathrm{T}}\left(A^{\mathrm{T}} P+P A\right) x=-x^{\mathrm{T}} R x=-L(x),
$$


which verifies (5) and (6). Hence

$$
J\left(x_{0}\right)=x_{0}^{\mathrm{T}} P x_{0} .
$$

Furthermore, since $V(x)$ is radially unbounded, the solution $x(t)=0, t \geqslant 0$, is, in this case, globally asymptotically stable. ${ }^{59}$ Alternatively, this result can be obtained more directly by using $x(t)=\mathrm{e}^{A t} x_{0}$ and

$$
J\left(x_{0}\right)=\int_{0}^{\infty}\left(\mathrm{e}^{A t} x_{0}\right)^{\mathrm{T}} R \mathrm{e}^{A t} x_{0} \mathrm{~d} t=x_{0} \int_{0}^{\infty} \mathrm{e}^{A^{T} t} R \mathrm{e}^{A t} \mathrm{~d} t x_{0}=x_{0}^{\mathrm{T}} P x_{0},
$$

where

$$
P=\int_{0}^{\infty} \mathrm{e}^{A^{T} t} R \mathrm{e}^{A t} \mathrm{~d} t
$$

is the unique, positive-definite solution to (12). Unfortunately, this technique does not generalize to the case of nonlinear dynamics.

\section{Remark 2.1}

Note that if (6) is valid, then (5) is equivalent to

$$
L(x)>0, \quad x \in \mathscr{D}, \quad x \neq 0 .
$$

More generally, assume $A$ is asymptotically stable, let $P$ be given by (12), and consider the case in which $L, f$, and $V$ are given by

$$
\begin{aligned}
& L(x)=x^{\mathrm{T}} R x+h(x), \\
& f(x)=A x+N(x), \\
& V(x)=x^{\mathrm{T}} P x+g(x),
\end{aligned}
$$

where $h: \mathscr{D} \rightarrow \mathbb{R}$ and $g: \mathscr{D} \rightarrow \mathbb{R}$ are nonquadratic and $N: \mathscr{D} \rightarrow \mathbb{R}^{n}$ is nonlinear. We assume that $g(\cdot)$ is $C^{1}$ so that $V(\cdot)$ is $C^{1}$. In this case (6) is satisfied if and only if

$$
x^{\mathrm{T}} R x+h(x)+\left[2 x^{\mathrm{T}} P+g^{\prime}(x)\right][A x+N(x)]=0, \quad x \in \mathscr{D},
$$

which can be rewritten as

$$
x^{\mathrm{T}}\left(A^{\mathrm{T}} P+P A+R\right) x+g^{\prime}(x) A x+h(x)+g^{\prime}(x) N(x)=0, \quad x \in \mathscr{D} .
$$

If $A$ is asymptotically stable, then we can (and will) choose $P$ to satisfy (12) as in the linear-quadratic case.

Next, suppose $N(x) \equiv 0$ and let $P$ satisfy (12). Then (9) becomes

$$
g^{\prime}(x) A x+h(x)=0, \quad x \in \mathscr{D} .
$$

Given $h(\cdot)$, our goal is to determine the existence of a $C^{1}$ function $g(\cdot)$ satisfying (20). To this end, we focus our attention on multilinear functions for which $(20)$ holds with $\mathscr{D}=\mathbb{R}^{n}$. Hence, let us call a function $\psi: \mathbb{R}^{n} \rightarrow \mathbb{R}^{p} k$-multilinear if $k$ is a positive integer and each component $\psi_{1}(x), \ldots, \psi_{p}(x)$ of $\psi(x)$ is a linear combination of terms of the form $x_{1}^{i_{1}} x_{2}^{i_{2}} \ldots x_{n}^{i_{n}}$, where $i_{j}$ is a nonnegative integer for $j=1, \ldots, n$, and $i_{1}+i_{2}+\cdots+i_{n}=k$. Clearly, $\psi(\cdot)$ satisfies $\psi(\alpha x)=\alpha^{k} \psi(x)$ for all $\alpha \in \mathbb{R}$ and $x \in \mathbb{R}^{n}$. Furthermore, a $k$-multilinear function $\psi: \mathbb{R}^{n} \rightarrow \mathbb{R}$ (where now $p=1$ ) is nonnegative definite (respectively, positive definite) if $\psi(x) \geqslant 0$ for all $x \in \mathbb{R}^{n}$ (respectively, $\psi(x)>0$ for all nonzero $x \in \mathbb{R}^{n}$ ). Obviously, if $k$ is odd then no 
$k$-multilinear function can be positive definite, while the only nonnegative-definite homogeneous $k$-form must be trivial.

If $\psi: \mathbb{R}^{n} \rightarrow \mathbb{R}^{p}$ is a $k$-multilinear function then $\psi$ can be represented concretely by means of Kronecker products, that is, $\psi(x)$ is given by

$$
\psi(x)=\Psi x^{[k]},
$$

where $\Psi \in \mathbb{R}^{p \times n^{k}}, x^{[k]} \triangleq x \otimes x \otimes \cdots \otimes x$ ( $k$ times), and $\otimes$ denotes Kronecker product. ${ }^{60}$ This representation for $\psi(x)$ is inefficient because of the repeated terms appearing in $x^{[k]}$. As shown in References 55, 61, the dimension $n^{k}$ can be reduced to

$$
\left(\begin{array}{c}
n+k-1 \\
k
\end{array}\right)
$$

We prefer to use Kronecker products, however, because of analytical convenience. Conversion to more compact notation can readily be carried out at a later stage to expedite numerical computation. The following lemma, which is quoted in Reference 38 and partially proved in Reference 46 will be useful for satisfying (20).

\section{Lemma 2.2}

Let $A \in \mathbb{R}^{n \times n}$ be asymptotically stable and let $h: \mathbb{R}^{n} \rightarrow \mathbb{R}$ be a $k$-multilinear function. Then there exists a unique $k$-multilinear function $g: \mathbb{R}^{n} \rightarrow \mathbb{R}$ such that

$$
g^{\prime}(x) A x+h(x)=0, \quad x \in \mathbb{R}^{n} .
$$

Furthermore, if $h(x)$ is nonnegative (respectively, positive) definite, then $g(x)$ is nonnegative (respectively, positive) definite.

Proof. For convenience define the repeated Kronecker sum

$$
\stackrel{k}{\oplus} A \triangleq A \oplus A \oplus \cdots \oplus A
$$

with $A$ appearing $k$ times, so that

$$
\begin{aligned}
& \stackrel{2}{\oplus} A=A \oplus A=A \otimes I+I \otimes A \\
& \stackrel{3}{\oplus} A=A \oplus A \oplus A=A \otimes I \otimes I+I \otimes A \otimes I+I \otimes I \otimes A
\end{aligned}
$$

and so forth. Let $h(x)=\Psi x^{[k]}$ and define $g(x) \triangleq \Gamma x^{[k]}$, where $\Gamma \triangleq-\Psi(\stackrel{k}{\oplus} A)^{-1}$, where $\stackrel{k}{\oplus} A$ is invertible since $A$ (and hence $\overbrace{}^{k} A$ ) is asymptotically stable. Now note that, for all $x \in \mathbb{R}^{n}$,

$$
\begin{aligned}
g^{\prime}(x) A x & =\Gamma \frac{\mathrm{d}}{\mathrm{d} x}\left(x^{[k]}\right) A x \\
& =\Gamma(A x \otimes x \otimes \cdots \otimes x+x \otimes A x \otimes \cdots \otimes x+\cdots+x \otimes x \otimes \cdots \otimes A x) \\
& =\Gamma(A \otimes I \otimes \cdots \otimes I+I \otimes A \otimes I \otimes \cdots \otimes I+\cdots+I \otimes I \otimes \cdots \otimes A) x^{[k]} \\
& =\Gamma(A \oplus A \oplus \cdots \oplus A) x^{[k]} \\
& =\Gamma\left(\kappa^{k} A\right) x^{[k]} \\
& =-\Psi x^{[k]} \\
& =-h(x) .
\end{aligned}
$$


To prove uniqueness, suppose that $\hat{g}(x)=\hat{\Gamma} x^{[k]}$ also satisfies (21). Then it follows that

$$
\Gamma(\stackrel{k}{\oplus} A) x^{[k]}=\hat{\Gamma}(\stackrel{k}{\oplus} A) x^{[k]}, \quad x \in \mathbb{R}^{n} .
$$

Since $\stackrel{k}{\oplus} A$ is asymptotically stable and $\mathrm{e}^{\left({ }^{k} \oplus A\right) t}=\left(\mathrm{e}^{A t}\right)^{[k]}$ (see Reference 60 for the case $k=2$ and Reference 62 for the case $k>2$ ), we have, for all $x \in \mathbb{R}^{n}$,

$$
\begin{aligned}
& \Gamma x^{[k]}=\Gamma(\stackrel{k}{\oplus} A)(\stackrel{k}{\oplus} A)^{-1} x^{[k]} \\
& =-\Gamma(\stackrel{k}{\oplus} A) \int_{0}^{\infty} \mathrm{e}^{(\stackrel{k}{\oplus} A) t} \mathrm{~d} t x^{[k]} \\
& =-\Gamma(\stackrel{k}{\oplus} A) \int_{0}^{\infty}\left(\mathrm{e}^{A t}\right)^{[k]} x^{[k]} \mathrm{d} t \\
& =-\int_{0}^{\infty} \Gamma(\stackrel{k}{\oplus} A)\left(\mathrm{e}^{A t} x\right)^{[k]} \mathrm{d} t \\
& =-\int_{0}^{\infty} \hat{\Gamma}(\stackrel{k}{\oplus} A)\left(\mathrm{e}^{A t} x\right)^{[k]} \mathrm{d} t \\
& =\hat{\Gamma} x^{[k]}
\end{aligned}
$$

which shows that $g(x)=\hat{g}(x), x \in \mathbb{R}^{n}$.

Finally, if $h(x)$ is nonnegative definite, then it follows that, for all $x \in \mathbb{R}^{n}$,

$$
\begin{aligned}
& g(x)=-\Psi(\stackrel{k}{\oplus} A)^{-1} x^{[k]} \\
& =\Psi \int_{0}^{\infty} \mathrm{e}^{(\stackrel{\kappa}{\oplus} A) t} \mathrm{~d} t x^{[k]} \\
& =\Psi \int_{0}^{\infty}\left(\mathrm{e}^{A t}\right)^{[k]} \mathrm{d} t x^{[k]} \\
& =\int_{0}^{\infty} \Psi\left(\mathrm{e}^{A t} x\right)^{[k]} \mathrm{d} t \\
& \geqslant 0 \text {. }
\end{aligned}
$$

If, in addition, $x \neq 0$, then $\mathrm{e}^{A t} x \neq 0, t \geqslant 0$. Hence if $h(x)$ is positive definite, then $g(x)$ is positive definite.

To illustrate Lemma 2.2, consider the linear system (10) and let $h(x)$ be a positive-definite $k$-multilinear function, where $k$ is necessarily even. Furthermore, let $g(x)$ be the positivedefinite $k$-multilinear function given by Lemma 2.2. Then, since $g^{\prime}(x) A x<0, x \in \mathbb{R}^{n}, x \neq 0$, it follows that $g(x)$ is a Lyapunov function for (10). Hence Lemma 2.10 can be used to generate Lyapunov functions of specific structure.

Suppose now that $h(x)$ in (15) is of the more general form

$$
h(x)=\sum_{\nu=2}^{r} h_{2 \nu}(x),
$$

where, for $\nu=2, \ldots, r, h_{2 \nu}: \mathbb{R}^{n} \rightarrow \mathbb{R}$ is a nonnegative-definite $2 \nu$-multilinear function. Now, using Lemma 2.2 , let $g_{2 v}: \mathbb{R}^{n} \rightarrow \mathbb{R}$ be the nonnegative-definite $2 \nu$-multilinear function satisfying

$$
g_{2 v}^{\prime}(x) A x+h_{2 v}(x)=0, \quad x \in \mathbb{R}^{n}, \quad \nu=2, \ldots, r,
$$


and define

$$
g(x)=\sum_{\nu=2}^{r} g_{2 v}(x)
$$

Since

$$
g^{\prime}(x)=\sum_{\nu=2}^{r} g_{2 v}^{\prime}(x)
$$

summing (23) over $v$ yields (21). Since (6) is now satisfied with $L(x)$ and $V(x)$ given by (15) and (17), result (7) implies that

$$
J\left(x_{0}\right)=x_{0}^{\mathrm{T}} P x_{0}+g\left(x_{0}\right)
$$

As another illustration of condition (20), suppose that $V(x)$ is constrained to be of the form

$$
V(x)=x^{\mathrm{T}} P x+\frac{1}{2}\left(x^{\mathrm{T}} M x\right)^{2},
$$

where $P$ satisfies (12) and $M$ is an $n \times n$ symmetric matrix. In this case $g(x)=\frac{1}{2}\left(x^{\mathrm{T}} M x\right)^{2}$ is a nonnegative-definite 4-multilinear function. Then (20) yields

$$
h(x)=-\left(x^{\mathrm{T}} M x\right) x^{\mathrm{T}}\left(A^{\mathrm{T}} M+M A\right) x .
$$

If $\hat{R}$ is an $n \times n$ symmetric matrix and $M$ is chosen to satisfy

$$
0=A^{\mathrm{T}} M+M A+\hat{R},
$$

then (27) implies that $h(x)$ satisfying (20) is of the form

$$
h(x)=\left(x^{\mathrm{T}} M x\right)\left(x^{\mathrm{T}} \hat{R} x\right) .
$$

Note that if $\hat{R}$ is nonnegative definite, then $M$ is also nonnegative definite and thus $h(x)$ is a nonnegative-definite 4-multilinear function. Thus, if $V(x)$ is of the form (26), and $L(x)$ is given by

$$
L(x)=x^{\mathrm{T}} R x+\left(x^{\mathrm{T}} M x\right)\left(x^{\mathrm{T}} \hat{R} x\right),
$$

where $M$ and $\hat{R}$ satisfy (28), then condition (20), and hence (6), is satisfied.

In Section 4 we apply Lemma 2.1 to the problem of optimal nonlinear feedback control. The relation (18) will play a key role with greater complexity arising from the fact that the nonlinear dynamics term $N(x)$ may be nonzero.

\section{OPTIMAL CONTROL WITH RESPECT TO A LYAPUNOV FUNCTION}

In this section we consider a control problem involving a notion of optimality that is directly related to a specified Lyapunov function. Although this control problem does not directly involve the optimization of a scalar cost functional, it does provide a transparent connection with the requirement of asymptotic stability while addressing explicit control constraints. These results also provide a natural prelude to the optimal control results in Section 4 .

To begin, let $\mathscr{D} \subset \mathbb{R}^{n}$ be an open set (as in Section 2) and let $\mathscr{U} \subset \mathbb{R}^{m}$ be an arbitrary set, where $0 \in \mathscr{D}$ and $0 \in \mathscr{U}$. Furthermore, let $F: \mathscr{D} \times \mathscr{U} \rightarrow \mathbb{R}^{n}$ satisfy $f(0,0)=0$. Now consider the controlled system

$$
\dot{x}(t)=f(x(t), u(t)), \quad x(0)=x_{0}, \quad t \geqslant 0 .
$$


The control $u(\cdot)$ in (31) is restricted to the class of admissible controls consisting of measurable functions $u(\cdot)$ such that

$$
u(t) \in \Omega, \quad t \geqslant 0,
$$

where the control constraint set $\Omega \subset \mathscr{U}$ is given. We assume that $0 \in \Omega$ and that $\Omega$ is compact.

A measurable mapping $\phi: \mathscr{D} \rightarrow \Omega$ satisfying $\phi(0)=0$ is called a control law. If $u(t)=\phi(x(t))$, where $\phi$ is a control law and $x(t)$ satisfies (31), then $u(\cdot)$ will be called a feedback control. Note that a feedback control is admissible since the control law $\phi(\cdot)$ takes values in $\Omega$. Given a control law $\phi(\cdot)$ and a feedback control $u(t)=\phi(x(t))$, the closed-loop system has the form

$$
\dot{x}(t)=f(x(t), \phi(x(t))), \quad x(0)=x_{0}, \quad t \geqslant 0 .
$$

Now consider a $C^{1}$ function $V: \mathscr{D} \rightarrow \mathbb{R}$ satisfying (3) and (4). The function $V$ will be a Lyapunov function for (31) if

$$
V^{\prime}(x(t)) f(x(t), u(t))<0, \quad t \geqslant 0,
$$

where $x(\cdot)$ is the solution to (31). Since $\dot{V}(x(t))=V^{\prime}(x(t)) f(x(t), u(t))$ represents the decay rate of $V(x(t))$, a reasonable criterion for choosing $u(t)$ is to minimize $\dot{V}(x(t))$. For each value of $x(t) \in \mathscr{D}$ the minimizing value of $u \in \Omega$ can be denoted by $\phi(x(t))$, thus leading to a (timeinvariant) control law $\phi: \mathscr{D} \rightarrow \Omega$. Note that this choice of control is made without regard for control effort except that $u(t) \in \Omega, t \geqslant 0$.

\section{Definition 3.1}

Let $V: \mathscr{D} \rightarrow \mathbb{R}$ be a $C^{1}$ function satisfying (3) and (4). Then the control law $\phi: \mathscr{D} \rightarrow \Omega$ is optimal with respect to $V$ if

$$
V^{\prime}(x) f(x, \phi(x)) \leqslant V^{\prime}(x) f(x, u), \quad x \in \mathscr{D}, \quad u \in \Omega .
$$

Thus, if $V$ satisfies (3), (4), and

$$
V^{\prime}(x) f(x, \phi(x))<0, \quad x \in \mathscr{D}, \quad x \neq 0,
$$

where $\phi: \mathscr{D} \rightarrow \Omega$, then $V$ is a Lyapunov function for the closed-loop system (33).

To illustrate Definition 3.1 consider the linear system

$$
\dot{x}(t)=A x(t)+B u(t), \quad x(0)=x_{0}, \quad t \geqslant 0 .
$$

For simplicity we assume that $A$ is asymptotically stable and define

$$
V(x)=x^{\mathrm{T}} P x,
$$

where, for some $n \times n$ positive-definite matrix $R$, the positive-definite matrix $P$ is the unique solution to

$$
0=A^{\mathrm{T}} P+P A+R
$$

Then it follows that

$$
V^{\prime}(x) f(x, u)=-x^{\mathrm{T}} R x+2\left(B^{\mathrm{T}} P x\right)^{\mathrm{T}} u, \quad x \in \mathscr{D}, \quad u \in \Omega .
$$

Thus the closed-loop system (33) is asymptotically stable, that is, (36) holds, if and only if

$$
2\left(B^{\mathrm{T}} P x\right)^{\mathrm{T}} \phi(x)<x^{\mathrm{T}} R x, \quad x \in \mathscr{D}, \quad x \neq 0 .
$$

Since $0 \in \Omega$ and $R$ is positive definite, (36) can always be satisfied by setting $\phi(x)=0$ which 
yields the asymptotically stable open-loop system. To choose $u=\phi(x)$ optimally with respect to $V$, however, we require (35), that is,

$$
\left(B^{\mathrm{T}} P x\right)^{\mathrm{T}} \phi(x) \leqslant\left(B^{\mathrm{T}} P x\right)^{\mathrm{T}} u, \quad x \in \mathscr{D}, \quad u \in \Omega .
$$

Since $\Omega$ is assumed to be compact, $\phi(x) \in \Omega$ satisfying (42) exists, although it need not be unique. In fact, if $B^{\mathrm{T}} P x=0$, then $\phi(x)$ is arbitrary. Since $u \rightarrow\left(B^{\mathrm{T}} P x\right)^{\mathrm{T}} u$ is linear, it follows from well-known results that if $\Omega$ is a convex polytope then $\phi(x)$ can be chosen to be a vertex of $\Omega$.

Now let $u(\cdot)$ be an admissible control such that $x(t) \rightarrow 0$ as $t \rightarrow \infty$, where $x(\cdot)$ satisfies (31). It thus follows that

$$
\begin{aligned}
V\left(x_{0}\right) & =V\left(x_{0}\right)-\lim _{t \rightarrow \infty} V(x(t)) \\
& =-\int_{0}^{\infty} \dot{V}(x(t)) \mathrm{d} t \\
& =-\int_{0}^{\infty} V^{\prime}(x(t)) f(x(t), u(t)) \mathrm{d} t \\
& =\int_{0}^{\infty}\left[x(t)^{\mathrm{T}} R x(t)+2\left(B^{\mathrm{T}} P x(t)\right)^{\mathrm{T}} u(t)\right] \mathrm{d} t .
\end{aligned}
$$

Defining the singular performance measure

$$
J\left(x_{0}, u(\cdot)\right) \triangleq \int_{0}^{\infty} x^{\mathrm{T}}(t) R x(t) \mathrm{d} t
$$

we obtain

$$
J\left(x_{0}, u(\cdot)\right)=V\left(x_{0}\right)-2 \int_{0}^{\infty}\left(B^{\mathrm{T}} P x(t)\right)^{\mathrm{T}} u(t) \mathrm{d} t,
$$

where $V\left(x_{0}\right)=x_{0}^{\mathrm{T}} P x_{0}$. Comparing (42) to (44), it is tempting to suggest that $u(\cdot)=\phi(x(\cdot))$ minimizes $J\left(x_{0}, u(\cdot)\right)$. This is false, however, since setting $u(\cdot)=\phi(x(\cdot))$ in (44) yields

$$
J\left(x_{0}, \phi(x(\cdot))\right)=V\left(x_{0}\right)-2 \int_{0}^{\infty}\left(B^{\mathrm{T}} P x(t)\right)^{\mathrm{T}} \phi(x(t)) \mathrm{d} t,
$$

where $x(t)$ inside the integral in (45) satisfies the closed-loop equation (33), whereas $x(t)$ in (44) satisfies (31). In fact, the optimal control for $J\left(x_{0}, u(\cdot)\right)$ is not that given by (42) but rather may involve singular arcs and time-varying gains. Furthermore, as noted in Section 4 , the case $B^{\mathrm{T}} P x=0$ is analogous to the singular case. For further details on this problem, see Reference 63 and references therein.

The above remarks can be illustrated by considering some special cases. First suppose that $\Omega$ is defined by

$$
\Omega \triangleq\{u:\|u\| \leqslant a\}
$$

where $\|\cdot\|$ denotes the Euclidean norm and $a>0$. Then a control law $\phi$ that is optimal with respect to $V$ is given by

$$
\phi(x)=\frac{-a}{\left\|B^{\mathrm{T}} P x\right\|} B^{\mathrm{T}} P x, \quad \text { if } B^{\mathrm{T}} P x \neq 0
$$


with $\phi(x)$ arbitrary if $B^{\mathrm{T}} P x=0$. In this case (45) becomes

$$
J\left(x_{0}, \phi(x(\cdot))\right)=x_{0}^{\mathrm{T}} P x_{0}-2 a \int_{0}^{\infty}\left\|B^{\mathrm{T}} P x(t)\right\| \mathrm{d} t .
$$

If, however, $\Omega$ is defined by

$$
\Omega \triangleq\left\{u=\left(u_{1}, \ldots, u_{m}\right)^{\mathrm{T}}:\left|u_{i}\right| \leqslant a_{i}, \quad i=1, \ldots, m\right\}
$$

for given positive constants $a_{1}, \ldots, a_{m}$, then it is easy to see that the control law $\phi=\left(\phi_{1}, \ldots, \phi_{m}\right)^{\mathrm{T}}: \mathbb{R}^{n} \rightarrow \Omega$ defined by

$$
\phi_{i}(x)=-a_{i} \operatorname{sgn}\left(B^{\mathrm{T}} P x\right)_{i}, \quad i=1, \ldots, m,
$$

is optimal with respect to $V$. Note that if $\left(B^{\mathrm{T}} P x\right)_{i}=0$, then $V^{\prime}(x) f(x, u)$ is independent of $u_{i}$ so that the value of $\phi_{i}(x)$ plays no role in (42). In this case (45) becomes

$$
J\left(x_{0}, \phi(x(\cdot))\right)=x_{0}^{\mathrm{T}} P x_{0}-2 \sum_{i=1}^{m} a_{i} \int_{0}^{\infty}\left|\left(B^{\mathrm{T}} P x(t)\right)_{i}\right| \mathrm{d} t .
$$

If, finally, one considers the constraint set

$$
\Omega \triangleq\left\{u=\left(u_{1}, \ldots, u_{m}\right)^{\mathrm{T}}: \sum_{i=1}^{m}\left|u_{i}\right| \leqslant a\right\}
$$

where $a>0$, then a control law $\phi$ that is optimal with respect to $V$ is given by

$$
\begin{aligned}
\phi_{i}(x) & =-a \operatorname{sgn}\left(B^{\mathrm{T}} P x\right)_{i}, \quad i=\mu\left(B^{\mathrm{T}} P x\right), \\
& =0, \quad \text { otherwise, }
\end{aligned}
$$

where $\mu\left(B^{\mathrm{T}} P x\right)$ denotes the index of the component of $B^{\mathrm{T}} P x$ having the largest absolute value. In this case (45) becomes

$$
J\left(x_{0}, \phi(x(\cdot))\right)=x_{0}^{\mathrm{T}} P x_{0}-2 a \int_{0}^{\infty}\left|\left(B^{\mathrm{T}} P x(t)\right)_{\mu\left(B^{\mathrm{T}} P x(t)\right)}\right| \mathrm{d} t .
$$

Now suppose that $A$ is not asymptotically stable. Then the stability condition (41) must be replaced by

$$
2\left(B^{\mathrm{T}} P x\right)^{\mathrm{T}} \phi(x)<-x^{\mathrm{T}}\left(A^{\mathrm{T}} P+P A\right) x, \quad x \in \mathscr{D}, \quad x \neq 0 .
$$

Since $A^{\mathrm{T}} P+P A$ cannot be negative definite, the expression $-x^{\mathrm{T}}\left(A^{\mathrm{T}} P+P A\right) x$ can assume arbtrarily large negative values if $\mathscr{D}=\mathbb{R}^{n}$. Thus, if $\Omega$ is compact, then at best only local asymptotic stability can be achieved, at least in the context of a quadratic Lyapunov function. To illustrate this case, consider $\Omega$ given by (46). Then with the control law (47), that is, the optimal control law with respect to $V$, condition (55) becomes

$$
x^{\mathrm{T}}\left(A^{\mathrm{T}} P+P A\right) x<2 a\left\|B^{\mathrm{T}} P x\right\|, \quad x \in \mathscr{D}, \quad x \neq 0 .
$$

In order to satisfy (56) it is clear that $x^{\mathrm{T}}\left(A^{\mathrm{T}} P+P A\right) x$ must be negative when $x$ lies in the null space of $B^{\mathrm{T}} P$. Furthermore, if $B^{\mathrm{T}} P X \neq 0$, then a sufficient condition for (56) is

$$
\frac{\|x\|^{2}}{\left\|B^{\mathrm{T}} P x\right\|}<\frac{2 a}{\bar{\sigma}\left(A^{\mathrm{T}} P+P A\right)}
$$

which provides a particular choice of $\mathscr{D}$, where $\bar{\sigma}$ denotes maximum singular value.

Note that each case considered has given rise to a nonlinear feedback control. This is not surprising in view of the hard saturation-type constraint on $u(t)$ given in the form of $\Omega$. In the 
next section we consider an optimal control problem in which $\Omega=\mathbb{R}^{n}$ but with a control magnitude penalty in the performance functional.

\section{OPTIMAL CONTROL}

In this section, we extend the development of Sections 2 and 3 to obtain a characterization of optimal feedback controllers. These conditions, which are a direct extension of Lemma 2.1, are essentially a specialization of the Hamilton-Jacobi-Bellman (HJB) conditions to the timeinvariant, infinite-horizon case. For this problem the HJB partial differential equation reduces to a purely algebraic relationship.

To address the problem of characterizing feedback controllers that minimize a performance functional, let $L: \mathbb{R}^{n} \times \mathbb{R}^{m} \rightarrow \mathbb{R}$ and, for $p \in \mathbb{R}^{n}$, define

$$
H(x, u, p) \triangleq L(x, u)+p^{\mathrm{T}} f(x, u) .
$$

Furthermore, define the set of asymptotically stabilizing controllers $\mathscr{S}\left(x_{0}\right)$ for each initial condition $x_{0} \in \mathscr{D}$, that is,

$\mathscr{S}\left(x_{0}\right) \triangleq\{u(\cdot): u(\cdot)$ is admissible and $x(\cdot)$ given by (3) satisfies $x(t) \rightarrow 0$ as $t \rightarrow \infty\}$.

\section{Theorem 3.1}

Consider the controlled system (31) with performance functional

$$
J\left(x_{0}, u(\cdot)\right) \triangleq \int_{0}^{\infty} L(x(t), u(t)) \mathrm{d} t
$$

Assume that there exist a $C^{1}$ function $V: \mathscr{D} \rightarrow \mathbb{R}$ and a control law $\phi: \mathscr{D} \rightarrow \Omega$ such that

$$
\begin{aligned}
V(0)=0 & & \\
V(x)>0, & x \in \mathscr{D}, & x \neq 0, \\
\phi(0)=0, & & \\
V^{\prime}(x) f(x, \phi(x))<0, & x \in \mathscr{D}, & x \neq 0, \\
H\left(x, \phi(x), V^{\prime \mathrm{T}}(x)\right)=0, & x \in \mathscr{D}, & \\
H\left(x, u, V^{\prime \mathrm{T}}(x)\right) \geqslant 0, & x \in \mathscr{D}, & u \in \Omega .
\end{aligned}
$$

Then, with the feedback control $u(\cdot)=\phi(x(\cdot))$, the solution $x(t)=0, t \geqslant 0$, of the closed-loop system (33) is locally asymptotically stable, and

$$
J\left(x_{0}, \phi(x(\cdot))\right)=V\left(x_{0}\right) .
$$

Furthermore, the feedback control $u(\cdot)=\phi(x(\cdot))$ minimizes $J\left(x_{0}, u(\cdot)\right)$ in the sense that

$$
J\left(x_{0}, \phi(x(\cdot))\right)=\min _{u(\cdot) \in \mathscr{F}\left(x_{0}\right)} J\left(x_{0}, u(\cdot)\right) .
$$

Proof. Local asymptotic stability is obtained by using (59)-(62) and applying Lemma 2.1 to the closed-loop system (33). Furthermore, using (63), condition (65) is a restatement of result (7) as applied to the closed-loop system. To prove (66), let $u(\cdot) \in \mathscr{S}\left(x_{0}\right)$ and let $x(\cdot)$ be the solution to (31). Then it follows that

$$
\dot{V}(x(t))=V^{\prime}(x(t)) f(x(t), u(t))
$$


or

$$
0=-\dot{V}(x(t))+V^{\prime}(x(t)) f(x(t), u(t))
$$

Hence

$$
\begin{aligned}
L(x(t), u(t)) & =-\dot{V}(x(t))+L(x(t), u(t))+V^{\prime}(x(t)) f(x(t), u(t)) \\
& =-\dot{V}(x(t))+H\left(x(t), u(t), V^{\prime \mathrm{T}}(x(t))\right) .
\end{aligned}
$$

Now using the fact that $u(\cdot) \in \mathscr{P}\left(x_{0}\right)$ along with (64) and (65), we obtain

$$
\begin{aligned}
J\left(x_{0}, u(\cdot)\right) & =\int_{0}^{\infty}\left[-\dot{V}(x(t))+H\left(x(t), u(t), V^{\prime \mathrm{T}}(x(t))\right)\right] \mathrm{d} t \\
& =-\lim _{t \rightarrow \infty} V(x(t))+V\left(x_{0}\right)+\int_{0}^{\infty} H\left(x(t), u(t), V^{\prime \mathrm{T}}(x(t))\right) \mathrm{d} t \\
& =V\left(x_{0}\right)+\int_{0}^{\infty} H\left(x(t), u(t), V^{\prime \mathrm{T}}(x(t))\right) \mathrm{d} t \\
& \geqslant V\left(x_{0}\right) \\
& =J\left(x_{0}, \phi(x(\cdot)),\right.
\end{aligned}
$$

which yields (66).

\section{Remark 4.1}

To relate Theorem 3.1 to the Hamilton-Jacobi-Bellman (HJB) equation, recall that the HJB equation is of the form

$$
\frac{\partial}{\partial t} V(t, x(t))+\min _{u \in \Omega} H\left(t, x(t), u, \frac{\partial}{\partial x} V(t, x(t))\right)=0
$$

which characterizes the optimal control for time-varying systems on a finite or infinite interval. In the time-invariant infinite-horizon case, $V$ is independent of $t$ and the HJB equation reduces to the algebraic (time-independent) relations (63) and (64).

\section{Remark 4.2}

Theorem 4.1 guarantees optimality with respect to the set $\mathscr{S}\left(x_{0}\right)$ of stabilizing admissible controls. Note, however, that an explicit characterization of $\mathscr{P}\left(x_{0}\right)$ is not required.

The principal feature of Theorem 3.1 is that the optimal control law $u=\phi(x)$ is a feedback controller. Furthermore, this control is stabilizing and its optimality is independent of the initial condition $x_{0}$.

\section{Remark 4.3.}

If (63) and (64) are satisfied, then it follows that

$$
L(x, \phi(x))+V^{\prime}(x) f(x, \phi(x)) \leqslant L(x, u)+V^{\prime}(x) f(x, u), \quad x \in \mathcal{D}, \quad u \in \Omega .
$$

If $L(x, u)$ is independent of $u$, then (67) is equivalent to

$$
V^{\prime}(x) f(x, \phi(x)) \leqslant V^{\prime}(x) f(x, u), \quad x \in \mathscr{D}, \quad u \in \Omega,
$$


which is precisely condition (35). Thus, in this case conditions (63) and (64) imply that the control law $\phi$ is optimal with respect to $V$. Thus, if the cost functional $J\left(x_{0}, u(\cdot)\right)$ is singular, then the optimal control laws characterized by Theorem 3.1 also optimize the decay rate as measured by the Lyapunov derivative. Note that this observation does not imply that the optimal control laws with respect to $V$ obtained in Section 3 are optimal in the sense of Theorem 3.1. This is due to the fact that condition (63) is not satisfied. For example, with $\Omega$ given by (46), $A$ asymptotically stable, $V$ given by (47), (48), and $J\left(x_{0}, u(\cdot)\right)$ defined by (43), it follows that

$$
H\left(x, \phi(x), V^{\prime}(x)\right)=-2 a\left\|B^{\mathrm{T}} P x\right\| .
$$

Thus we can now see more clearly that the controllers given in Section 3 do not, in general, optimize $J\left(x_{0}, u(\cdot)\right)$ defined by (33) because the Lyapunov function $V(x)=x^{\mathrm{T}} P x$ is not a solution of the HJB equation. If, however, $B^{\mathrm{T}} P x=0$, then (63) is satisfied. Since the adjoint state $p(t)$ satisfying the maximum principle is given by $-V^{\prime}(x(t))$ where $V(x(t))$ satisfies the HJB equation, ${ }^{64}$ it can be seen that $B^{\mathrm{T}} P x(t)=0$ corresponds to $p^{\mathrm{T}}(t) B=0$. This accounts for our interpretation of the case $B^{\mathrm{T}} P x=0$ as a singular condition.

Now let us illustrate Theorem 3.1 with a simple example. We begin with the simplest case, namely, the linear-quadratic regulator. Hence consider the controlled system

$$
\dot{x}=A x+B u, \quad x(0)=x_{0}, \quad t \geqslant 0,
$$

with performance functional

$$
J\left(x_{0}, u(\cdot)\right)=\int_{0}^{\infty}\left[x^{\mathrm{T}} R_{1} x+u^{\mathrm{T}} R_{2} u\right] \mathrm{d} t,
$$

where $x \in \mathscr{D}=\mathbb{R}^{n}, u \in \Omega=\mathscr{U}=\mathbb{R}^{m}$, and where $R_{1}$ and $R_{2}$ are positive definite. We shall let $V(x)$ be quadratic as in Section 3, that is,

$$
V(x)=x^{\mathrm{T}} P x,
$$

where $P$ is positive definite. Thus the stability condition (62) is satisfied by the control law $\phi$ if

$$
2\left(B^{\mathrm{T}} P x\right)^{\mathrm{T}} \phi(x)<-x^{\mathrm{T}}\left(A^{\mathrm{T}} P+P A\right) x, \quad x \in \mathbb{R}^{n}, \quad x \neq 0 .
$$

Furthermore, we have

$$
H\left(x, V^{\prime}(x), u\right)=x^{\mathrm{T}}\left(A^{\mathrm{T}} P+P A+R_{1}\right) x+2 x^{\mathrm{T}} P B u+u^{\mathrm{T}} R_{2} u .
$$

To determine $u=\phi(x)$ that minimizes $H\left(x, u, V^{\prime} \mathrm{T}(x)\right)$ and satisfies (63), we can seek the global minimum of $H\left(x, u, V^{\prime}(x)\right)$. This approach is permissible since $\Omega=\mathbb{R}^{\mathrm{m}}$. Hence setting

$$
\frac{\partial}{\partial u} H\left(x, u, V^{\prime \mathrm{T}}(x)\right)=0
$$

yields the control

$$
\phi(x)=-R_{2}^{-1} B^{\mathrm{T}} P x .
$$

Since

$$
\begin{gathered}
\frac{\partial^{2}}{\partial u^{2}} H\left(x, u, V^{\prime} \mathrm{T}(x)\right)=R_{2}>0 \\
\text { it follows that for each } x \in \mathbb{R}^{n} \text { the control law (75) minimizes the mapping }
\end{gathered}
$$


$u \rightarrow H\left(x, u, V^{\prime} \mathrm{T}(x)\right)$. With (76) it follows that

$$
H\left(x, \phi(x), V^{\prime} \mathrm{T}(x)\right)=x^{\mathrm{T}}\left(A^{\mathrm{T}} P+P A+R_{1}-P S P\right) x, \quad x \in \mathbb{R}^{n},
$$

where $S \triangleq B R_{2}^{-1} B^{\mathrm{T}}$. Thus, (63) holds if and only if $P$ is chosen to satisfy the algebraic Riccati equation

$$
0=A^{\mathrm{T}} P+P A+R_{1}-P S P .
$$

In this case the stability condition (73) becomes

$$
0<x^{\mathrm{T}}\left(P S P+R_{1}\right) x,
$$

which is satisfied since $R_{1}$ is positive definite and $S$ is nonnegative definite. The closed-loop system now has the form

$$
\dot{x}=(A-S P) x, \quad x(0)=x_{0}, \quad t \geqslant 0,
$$

while the optimal cost is given by

$$
J\left(x_{0}, \phi(x(\cdot))\right)=x_{0}^{\mathrm{T}} P x_{0} .
$$

Note that (79) can also be written as

$$
0=(A-S P)^{\mathrm{T}} P+P(A-S P)+R_{1}+P S P,
$$

which shows that $A-S P$ is asymptotically stable. Finally, it can be seen that

$$
H\left(x, u, V^{\prime} \mathrm{T}(x)\right)=[u-\phi(x)]^{\mathrm{T}} R_{2}[u-\phi(x)],
$$

which renders (63) and (64) immediate.

In the next section we use the framework of Theorem 4.1 to consider optimal control problems involving nonquadratic performance measures.

\section{OPTIMAL CONTROL WITH NONQUADRATIC COST}

In this section we continue to illustrate Theorem 4.1 by applying it to optimal control problems involving nonquadratic performance measures. The results obtained are closely related to the nonlinear feedback controllers derived in References 38 and 45. As noted in Section 1, the main goal of this paper is to unify and clarify these results.

Hence assume that $L(x, u), f(x, u)$, and $V(x)$ are of the form

$$
\begin{aligned}
L(x, u) & =x^{\mathrm{T}} R_{1} x+h(x)+u^{\mathrm{T}} R_{2} u, \\
f(x, u) & =A x+B u, \\
V(x) & =x^{\mathrm{T}} P x+g(x),
\end{aligned}
$$

where $h: \mathscr{D} \rightarrow \mathbb{R}$ and where $g: \mathscr{D} \rightarrow \mathbb{R}$ is $C^{1}$ and satisfies $g(x) \geqslant 0, x \in \mathscr{D}$. With this notation it follows that

$$
H\left(x, u, V^{\prime \mathrm{T}}(x)\right)=x^{\mathrm{T}} R_{1} x+h(x)+u^{\mathrm{T}} R_{2} u+\left[2 x^{\mathrm{T}} P+g^{\prime}(x)\right][A x+B u] .
$$

Setting $(\partial / \partial u) H\left(x, u, V^{\prime} \mathrm{T}(x)\right)=0$ yields the control law

$$
\phi(x)=\frac{1}{2} R_{2}^{-1} B^{\mathrm{T}} V^{\prime \mathrm{T}}(x)=-R_{2}^{-1} B^{\mathrm{T}} P x-\frac{1}{2} R_{2}^{-1} B^{\mathrm{T}} g^{\prime \mathrm{T}}(x)
$$

which minimizes $H\left(x, u, V^{\prime} \mathrm{T}(x)\right)$ because of (76). With (89) it follows that

$$
\begin{aligned}
V^{\prime}(x) f(x, \phi(x))=x^{\mathrm{T}}\left(A^{\mathrm{T}} P+P A-2 P S P\right) x-x^{\mathrm{T}} P S g^{\prime \mathrm{T}}(x) \\
+g^{\prime}(x)(A-S P) x-\frac{1}{2} g^{\prime}(x) S g^{\prime \mathrm{T}}(x), \quad x \in \mathscr{D},
\end{aligned}
$$


and thus

$H\left(x, \phi(x), V^{\prime} \mathrm{T}(x)\right)=x^{\mathrm{T}}\left(A^{\mathrm{T}} P+P A+R_{1}-P S P\right) x+g^{\prime}(x)(A-S P) x+h(x)-\frac{1}{4} g^{\prime}(x) S g^{\prime \mathrm{T}}(x)$.

To satisfy (63), let $P$ satisfy (78) and require that

$$
g^{\prime}(x)(A-S P) x+h(x)-\frac{1}{4} g^{\prime}(x) S g^{\prime \mathrm{T}}(x)=0, \quad x \in \mathscr{D} .
$$

In this case $(90)$ becomes

$$
V^{\prime}(x) f(x, \phi(x))=-\left[x^{\mathrm{T}} R_{1} x+h(x)+\left(x^{\mathrm{T}} P+\frac{1}{2} g^{\prime}(x)\right) S\left(P x+\frac{1}{2} g^{\prime \mathrm{T}}(x)\right)\right], \quad x \in \mathscr{D},
$$

which verifies the stability condition (62) if $h(x) \geqslant 0, x \in \mathscr{D}$. Furthermore, with $\phi(x)$ given by (89), it can be shown that

$$
H\left(x, u, V^{\prime}{ }^{\mathrm{T}}(x)\right)=[u-\phi(x)]^{\mathrm{T}} R_{2}[u-\phi(x)],
$$

which verifies (64). We have yet to determine $g(x)$ and verify the stability condition (62).

Returning to (92), let us consider the approach of Reference 38. Suppose that for $\nu=2, \ldots, r, h_{2 \nu}(x)$ is a given nonnegative-definite $2 \nu$-multilinear function. Since $A-S P$ is asymptotically stable, Lemma 2.2 implies that, for $\nu=2, \ldots, r$, there exists a unique nonnegative-definite $2 \nu$-multilinear function $g_{2 \nu}(x)$ satisfying

$$
g_{2 \nu}^{\prime}(x)(A-S P) x+h_{2 \nu}(x)=0, \quad \nu=2, \ldots, r .
$$

Then (92) can be satisfied with $h(x)$ and $g(x)$ defined by

$$
\begin{aligned}
& h(x)=\sum_{\nu=2}^{r} h_{2 \nu}(x)+\frac{1}{4} g^{\prime}(x) S g^{\prime \mathrm{T}}(x), \\
& g(x)=\sum_{\nu=2}^{r} g_{2 v}(x) .
\end{aligned}
$$

As discussed in Reference 38, the term $\frac{1}{4} g^{\prime}(x) S g^{\prime T}(x)$ appearing in $h(x)$ in (96) and hence in the cost functional (58) via (85) is somewhat artificial in the sense that it cannot be specified arbitrarily. It is interesting to note, however, that with $h(x)$ given by (85), the performance functional has the form

$$
J\left(x_{0}, u(\cdot)\right)=\int_{0}^{\infty}\left[x^{\mathrm{T}} R_{1} x+\sum_{\nu=2}^{r} h_{2 v}(x)+u^{\mathrm{T}} R_{2} u+\phi_{\mathrm{NL}}^{\mathrm{T}}(x) R_{2} \phi_{\mathrm{NL}}(x)\right] \mathrm{d} t .
$$

In (98), $\phi_{\mathrm{NL}}(x)$ is the nonlinear part of the optimal feedback control (89), that is,

$$
\phi(x)=\phi_{\mathrm{L}}(x)+\phi_{\mathrm{NL}}(x),
$$

where

$$
\phi_{\mathrm{L}}(x) \triangleq-R_{2}^{-1} B^{\mathrm{T}} P x, \quad \phi_{\mathrm{NL}}(x) \triangleq-\frac{1}{2} R_{2}^{-1} B^{\mathrm{T}} g^{\prime \mathrm{T}}(x) .
$$

As another example, we consider a deterministic version of the stochastic nonlinear-nonquadratic optimal control problem considered in Reference 45 . Hence we require that $V(x)$ be of the form

$$
V(x)=x^{\mathrm{T}} P x+\frac{1}{2}\left(x^{\mathrm{T}} M x\right)^{2}
$$

which corresponds to (87) with $g(x)=\frac{1}{2}\left(x^{\mathrm{T}} M x\right)^{2}$. Thus (89) specializes to the control law

$$
\phi(x)=-R_{2}^{-1} B^{\mathrm{T}} P x-R_{2}^{-1} B^{\mathrm{T}}\left(x^{\mathrm{T}} M x\right) M x,
$$


and, with $P$ given by (78), condition (92) becomes

$$
h(x)+\left(x^{\mathrm{T}} M x\right) x^{\mathrm{T}}\left[(A-S P)^{\mathrm{T}} M+M(A-S P)\right] x-\left(x^{\mathrm{T}} M x\right)^{2} x^{\mathrm{T}} M S M x=0 .
$$

To satisfy (103), let $R$ be an arbitrary $n \times n$ symmetric matrix and, since $A-S P$ is asymptotically stable, let $M$ be the unique, symmetric solution to

$$
(A-S P)^{\mathrm{T}} M+M(A-S P)+R=0
$$

Now (103) is satisfied with $h(x)$ chosen to be

$$
h(x)=\left(x^{\mathrm{T}} M x\right)\left(x^{\mathrm{T}} R x\right)+\left(x^{\mathrm{T}} M x\right)^{2} x^{\mathrm{T}} M S M x .
$$

Again, as in Reference 38, the cost functional cannot be specified arbitrarily since the matrix $M$ depends upon $P$. In Reference 45, $M$ was chosen to satisfy the Riccati equation

$$
(A-S P)^{\mathrm{T}} M+M(A-S P)+R+M S M=0
$$

which can be seen as equation (23) of Reference 45 with $w_{2}=0, F=1$, and obvious changes of notation. With this choice of $M, h(x)$ is given by

$$
h(x)=\left(x^{\mathrm{T}} M x\right)\left(x^{\mathrm{T}} R x\right)+\left(x^{\mathrm{T}} M x\right)\left(x^{\mathrm{T}} M S M x\right)+\left(x^{\mathrm{T}} M x\right)^{2} x^{\mathrm{T}} M S M x
$$

which is given by (4) of Reference 45. Note that the choice of (104) for $M$ simplifies the result of Reference 45 since (104) is linear and (105) involves only one quartic term.

\section{CONCLUSIONS}

This paper has presented a tutorial exposition and simplified framework for some results on optimal nonlinear regulation with nonquadratic cost functionals. Considering time-invariant systems on the infinite interval, we utilized a steady-state Hamilton-Jacobi-Bellman approach to characterize optimal nonlinear feedback controllers. To illustrate the approach, we rederived the classical results of Bass and Webber ${ }^{38}$ and obtained a deterministic variation of the stochastic nonlinear feedback controller given by Speyer. ${ }^{45}$ Besides clarifying and simplifying these ideas as developed by prior researchers, this exposition is intended to provide a framework for fixed-structure nonlinear-nonquadratic control, which is currently under development.

\section{ACKNOWLEDGEMENT}

I wish to thank J. O'Sullivan, E. G. Gilbert, D. C. Hyland and E. G. Collins, Jr, for helpful discussions.

\section{REFERENCES}

1. Kwakernaak, H., and R. Sivan, Linear Optimal Control Systems, Wiley, New York, 1972.

2. Meyer, G., R. Su, and L. R. Hunt, 'Application of nonlinear transformations to automatic flight control', Automatica, 20, 103-107 (1984).

3. Isidori, A., Nonlinear Control Systems: An Introduction, 2nd edn, Springer-Verlag, New York, 1989.

4. Baumann, W. T., and W. J. Rugh, 'Feedback control of nonlinear systems by extended linearization', IEEE Trans. Autom. Contr., AC-31, 40-46 (1986).

5. Wang, J., and W. J. Rugh, 'Feedback linearization families for nonlinear systems', IEEE Trans. Autom. Contr., AC-32, 935-941 (1987).

6. Shamma, J. S., and M. Athans, 'Analysis of gain scheduled control for nonlinear plants', IEEE Trans. Autom. Contr., AC-35, 898-907 (1990). 
7. Khargonekar, P. P., and K. R. Poolla, 'Uniformly optimal control of linear time-invariant plants: nonlinear timeinvariant controllers', Sys. Contr. Lett., 6, 303-308 (1986).

8. Khargonekar, P. P., T. T. Georgiou, and A. M. Pascoal, 'On the robust stabilizability of linear time-invariant plants with unstructured uncertainty', IEEE Trans. Autom. Contr., AC-32, 201-207 (1987).

9. Gutman, S., 'Uncertain dynamical systems - a Lyapunov min-max approach', IEEE Trans. Autom. Contr., AC-24, 437-443 (1979).

10. Barmish, B. R., 'Stabilization of uncertain systems via linear control', IEEE Trans. Autom. Contr., AC-28, $848-850$ (1983).

11. Petersen, I. R., 'Nonlinear versus linear control in the direct output feedback stabilization of linear systems', IEEE Trans. Autom. Contr., AC-30, 799-802 (1985).

12. Petersen, I. R., 'Quadratic stabilizability of uncertain linear systems: existence of a nonlinear stabilizing control does not imply existence of a linear stabilizing control', IEEE Trans. Autom. Contr., AC-30, 291-293 (1985).

13. Barmish, B. R., and A. R. Galimidi, 'Robustness of Luenberger observers: linear systems stabilized via non-linear control', Automatica, 22, 413-423 (1986).

14. Leitmann, G., 'On the efficacy of nonlinear control in uncertain linear systems', J. Dyn. Sys. Meas. Contr., 102, 95-102 (1981).

15. Corless, M. J., and G. Leitmann, 'Continuous state feedback guaranteeing uniform ultimate boundedness for uncertain dynamic systems', IEEE Trans. Autom. Contr., AC-26, 1139-1144 (1981).

16. Itkis, U., Control Systems of Variable Structure, Wiley, New York, 1976.

17. Utkin, V. I., 'Variable structure systems with sliding modes', IEEE Trans. Autom. Contr., AC-22, 212-222 (1977).

18. Slotine, J. J. E., 'Sliding controller design for nonlinear systems', Int. J. Contr., 40, 421-434 (1984).

19. Ryan, E. P., 'A variable structure approach to feedback regulation of uncertain systems', Int. J. Contr., 38, 1121-1134 (1983).

20. Basar, T., 'Disturbance attenuation in LTI plants with finite horizon: optimality of nonlinear controllers', Sys. Contr. Lett., 13, 183-191 (1989).

21. Nussbaum, R. D., 'Some remarks on a conjecture in adaptive control', Sys. Contr. Lett., 6, 87-91 (1985).

22. Martensson, B., 'The order of any stabilizing regulator is sufficient information for adaptive stabilization', Sys. Contr. Lett., 6, 87-91 (1985).

23. Mudgett, D. R., and A. S. Morse, 'Adaptive stabilization of linear systems with unknown high-frequency gains', IEEE Trans. Autom. Contr., AC-30, 549-554 (1985).

24. Fu, M., and B. R. Barmish, 'Adaptive stabilization of linear systems via switching control', IEEE Trans. Autom. Contr., AC-31, 1097-1103 (1986).

25. Frankena, J. F., and R. Sivan, 'A nonlinear optimal control law for linear systems', Int. J. Contr., 30, 159-178 (1979).

26. Ryan, E. P., 'Optimal feedback control of saturating systems', Int. J. Contr., 35, 531-534 (1982).

27. Kosut, R. L., 'Design of linear systems with saturating linear control and bounded states', IEEE Trans. Autom. Contr., AC-28, 121-124 (1983).

28. Gutman, P.-O., 'A new design of constrained controllers for linear systems', IEEE Trans. Autom. Contr., AC-30, 22-33 (1985).

29. Hanus, R., M. Kinnaert, and J.-L. Henrotte, "Conditioning technique, a general anti-windup and bumpless transfer method', Automatica, 23, 729-739 (1987).

30. Keerthi, S. S., and E. G. Gilbert, 'Computation of minimum-time feedback control laws for discrete-time systems with state-control constraints', IEEE Trans. Autom. Contr., AC-32, 432-435 (1987).

31. Blanchini, F., 'Feedback control for linear time-invariant systems with state and control bounds in the presence of disturbances', IEEE Trans. Autom. Contr., 35, 1231-1234 (1990).

32. Sontag, E. D., and H. J. Sussmann, 'Nonlinear output feedback design for linear systems with saturating controls', Proc. Conf. Dec. Contr., pp. 3414-3416, Honolulu, HI, December 1990.

33. Wilson, D. A., 'Convolution and Hankel operator norms for linear systems', IEEE Trans. Autom. Contr., AC-34, 94-97 (1989).

34. Rotea, M., 'Generalized $\mathrm{H}_{2} / \mathrm{H}_{\infty}$ control via convex optimization', Proc. Conf. Dec. Contr., pp. 2719-2720, Brighton, UK, December 1991.

35. Kalman, R. E., 'The theory of optimal control and the calculus of variations', in Mathematical Optimization Techniques, R. Bellman (ed.), University of California Press, Berkeley, CA, 1963.

36. Rekasius, Z. V., 'Suboptimal design of intentionally nonlinear controllers', IEEE Trans. Autom. Contr., AC-9, 380-386 (1964).

37. Al'brekht, E. G., 'The existence of an optimal Lyapunov function and of a continuous optimal control for one problem on the analytical design of controllers', Differentsial'nye Uravneniya, 1, 1301-1311 (1965).

38. Bass, R. W., and R. F. Webber, 'Optimal nonlinear feedback control derived from quartic and higher-order performance criteria', IEEE Trans. Autom. Contr., AC-11, 448-454 (1966).

39. Garrard, W. L., N. H. McClamroch, and L. G. Clark, 'An approach to suboptimal feedback control of nonlinear systems', Int. J. Contr., 5, 425-435 (1967).

40. Lukes, D. L., 'Optimal regulation of nonlinear dynamical systems', SIAM J. Contr., 9, 87-88 (1971). 
41. Thau, F. E., 'Optimum nonlinear control of a class of randomly excited systems', J. Dyn. Sys. Meas. Contr., March, 41-44 (1971).

42. Rugh, W. J., 'System equivalence in a class of nonlinear optimal control problems', IEEE Trans. Autom. Contr., AC-16, 189-194 (1971).

43. Moylan, P. J., and B. D. O. Anderson, 'Nonlinear regulator theory and an inverse optimal control problem', IEEE Trans. Autom. Contr., AC-18, 460-465 (1973).

44. Halme, A., and R. Hamalainen, 'On the nonlinear regulator problem', J. Optim. Thy. Appl., 16, 255-275 (1975).

45. Speyer, J. L., 'A nonlinear control law for a stochastic infinite time problem', IEEE Trans. Autom. Contr., AC-21, 560-564 (1976).

46. Sandor, J., and D. Williamson, 'Design of nonlinear regulators for linear plants', IEEE Trans. Autom. Contr., AC-22, 47-50 (1977).

47. Willemstein, A. P., 'Optimal regulation of nonlinear dynamical systems on a finite interval', SIAM J. Contr., 15, 1050-1069, 1977.

48. Baillieul, J., 'Multilinear optimal control', Proc. Conference on Geometry for the Control Engineer, pp. 337-359, Math. Sci. Press, Brookline, MA, 1977.

49. Shaw, L., 'Nonlinear control of linear multivariable systems via state-dependent feedback gains', IEEE Trans. Autom. Contr., AC-24, 108-112 (1979).

50. Salehi, S. V., and E. P. Ryan, 'On optimal nonlinear feedback regulation of linear plants', IEEE Trans. Autom. Contr., AC-27, 1260-1264 (1982).

51. Beaman, J. J., 'Nonlinear quadratic Gaussian control', Int. J. Contr., 39, 343-361 (1984).

52. Ozgoren, M. K., and R. W. Longman, 'Automated derivation of optimal regulators for nonlinear systems by symbolic manipulation of Poisson series', J. Optim. Thy. Appl., 45, 443-476 (1985).

53. O'Sullivan, J. A., 'Nonlinear optimal regulation by polynomic approximation methods', Ph.D. Dissertation, University of Notre Dame, Notre Dame, IN, 1986.

54. Rouff, M., and F. Lamnabhi-Lagarrigue, 'A new approach to nonlinear optimal feedback law', Sys. Contr. Lett., 7, 411-417 (1986)

55. Hopkins, W. E. Jr, 'Optimal linear control of single-input nonlinear systems', Proc. Amer. Contr. Conf., pp. 1981-1983, Minneapolis, MN, June 1987.

56. Bourdache-Siguerdidjane, H., and M. Fliess, 'Optimal feedback control of nonlinear systems', Automatica, 23, 365-372 (1987).

57. Rotella, F., and G. Dauphin-Tanguy, 'Non-linear systems: identification and optimal control', Int. J. Contr., 48 525-544 (1988).

58. Yoshida, T., and K. A. Loparo, 'Quadratic regulatory theory for analytic nonlinear systems with additive controls', Automatica, 25, 531-544 (1989).

59. Vidyasagar, M., Nonlinear Systems Theory, Prentice Hall, Englewood Cliffs, NJ, 1978.

60. Brewer, J. W. 'Kronecker products and matrix calculus in system theory', IEEE Trans. Circ. Sys., 25, 772-781 (1978).

61. Brockett, R. W., 'Volterra series and geometric control theory', Automatica, 12, 167-176 (1976)

62. Steeb, W.-H., and F. Wilhelm, 'Exponential functions of Kronecker products and trace calculation', Lin. Multilinear Alg., 9, 345-346 (1981).

63. Bernstein, D. S., and V. Zeidan, 'The singular linear-quadratic regulator problem and the Goh-Riccati equation', Proc. Conf. Dec. Contr., pp. 334-339, Honolulu, HI, December 1990.

64. Clarke, F. H., and R. B. Vinter, 'The relationship between the maximum principle and dynamic programming', SIAM J. Contr. Optim., 25, 1291-1311 (1987). 


\section{ERRATUM}

Nonquadratic Cost and Noninear Feedback Control, 3, 211-229, 1993.

Page 213, line 1. Change ' 3.1 ' to ' 4.1 '.

Page 214, line 1. Append 'there exists a neighborhood of the origin $\mathscr{D}_{0} \subset \mathscr{D}$ such that if $x_{0} \in \mathscr{D}_{0}$ then'.

Page 214, line 2. Delete ', $x_{0} \in \mathscr{D}$ '.

Page 214, line 9. Change ' $D$ ' to ' $\mathscr{D}_{0}$ for some neighborhood of the origin $\mathscr{D}_{0} \subset \mathscr{D}$ '.

Page 214 , line 12. Change ' $\dot{V}$ to ' $-\dot{V}$ '.

Page 214 , line 16. Change ' $D$ to ' $D_{0}$ '.

Page 219, line 4. Delete 'and that $\Omega$ is compact'.

Page 220, line 4. Change 'Since' to 'If'.

Page 220, line 13. Change ' + ' to '-'.

Page 220, line 17. Change ' - ' to ' + '.

Page 220, line 20. Change ' - ' to ' + '.

Page 222, line 13. Change ' $\in \mathscr{D}$ ' to 'contained in a neighborhood $\mathscr{D}_{0} \subset \mathscr{D}$ '.

Page 222, line 15 Change ' 3.1 ' to ' 4.1 '.

Page 222, line 26. Append 'there exists a neighborhood of the origin $\mathscr{D}_{0} \subset \mathscr{D}$ such that, if $x_{0} \in \mathscr{D}_{0}$, then'.

Page 222, line 28. Append 'if $x_{0} \in \mathscr{D}_{0}$, then'.

Page 223, lines 14 and 23 . Change ' 3.1 ' to ' 4.1 '.

Page 224, lines 3, 6, and 16 . Change ' 3.1 ' to ' 4.1 '.

Page 224, line 7. Change '(47), (48)' to '(38)'.

Page 224, line 11. Change '(33)' to '(43)'.

Page 224, line 35. Change '(75)' to '(76)'.

Page 225, line 34. Change '(76)' to '(77)'.

Page 226, line 3. Change '(78)' to '(79)'.

Page 226, line 21. Change '(85)' to '(96)'.

Page 227, line 1. Change '(78)' to '(79)'.

Dennis S. Bernstein 